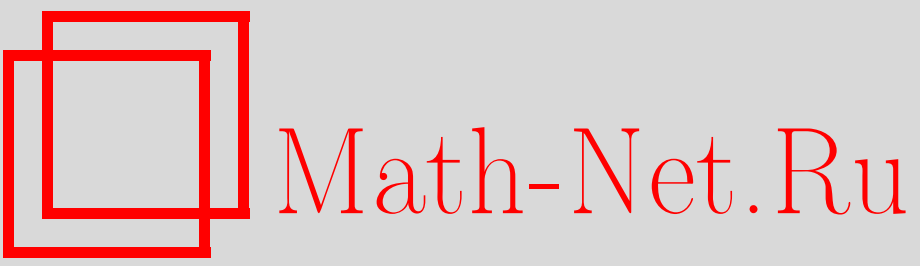

А. Р. Бикметов, Д. И. Борисов, О дискретном спектре оператора Шредингера, возмущенного ограниченным потенциалом с малым носителем, ТM $\Phi, 2005$, том 145, номер 3, 372-384

DOI: https://doi.org/10.4213/tmf1906

Использование Общероссийского математического портала Math-Net.Ru подразумевает, что вы прочитали и согласны с пользовательским соглашением

http: //www . mathnet.ru/rus/agreement

Параметры загрузки:

IP : 35.173 .219 .149

26 апреля 2023 г., 15:55:06 


\title{
О ДИСКРЕТНОМ СПЕКТРЕ ОПЕРАТОРА ШРЕДИНГЕРА, ВОЗМУШЕННОГО ОГРАНИЧЕННЫМ ПОТЕНЦИАЛОМ С МАЛЫМ НОСИТЕЛЕМ
}

\begin{abstract}
Изучаются асимптотические свойства дискретного спектра оператора Шредингера, возмущенного потенциалом с узким носителем. Для собственных значений и соответствующих собственных функций строятся первые члены асимптотических разложений по малому параметру, которым является ширина носителя потенциала.
\end{abstract}

Ключевые слова: оператор Шредингера, спектр, возмущение, асимптотика.

\section{1. ВВЕДЕНИЕ}

Изучению дискретного спектра оператора Шредингера с различного рода возмушениями посвящено достаточно много работ (см., например, [1]-[5]). Классической задачей является исследование возмушения стационарного оператора Шредингера на оси и плоскости малым потенциалом вида $\varepsilon V(x), 0<\varepsilon \ll 1, \int_{\mathbb{R}}(1+|x|)|V(x)| d x<\infty$, что соответствует малой (неглубокой) потенциальной яме либо барьеру, в зависимости от знака $V$. Такое возмушение рассматривалось в $§ 45$ книге [1] и в работах [2]. Случай нелинейной зависимости потенциала от малого параметра был рассмотрен в [4]. В работах [5] результаты, полученные в работах [1]-[4], были обобщены на случай возмущения вида $\varepsilon L_{\varepsilon}$, где $L_{\varepsilon}: W_{2}^{1}(Q) \rightarrow L_{2}(Q)$ - произвольный оператор, ограниченный равномерно по $\varepsilon, Q$ - произвольная ограниченная область в $\mathbb{R}^{n}, n=1,2$, а функции из $L_{2}(Q)$ считаются продолженными нулем вне $Q$.

Настоящая работа посвящена изучению иного случая, когда возмушение описывается потенциалом, принимаюшим конечные (не малые) значения, но имеющим малый носитель. А именно, изучается дискретный спектр оператора

$$
\mathcal{H}_{\varepsilon}:=-\Delta+V\left(\frac{x-x_{0}}{\varepsilon}\right)
$$

в ограниченной односвязной области $\Omega \subset \mathbb{R}^{n}, n=1,2,3$, с граничным условием Дирихле на границе. В случае $n=1$ мы полагаем $\Omega=(0,1), \Delta=d^{2} / d x^{2}$, в случае $n=2,3$

* Башкирский государственный педагогический университет, Уфа, Россия. E-mail: BikmetovAR@bspu.ru, BorisovDI@ic.bashedu.ru 
граница области $\Omega$ предполагается бесконечно дифференцируемой. Функция $V(t)$ является произвольной вешественнозначной бесконечно дифференшируемой финитной функцией, определенной на всем пространстве, $x_{0}$ - некоторая фиксированная точка из $\Omega$. Так как функция $V$ финитна, то носитель потенциала в (1) сжимается в точку $x_{0}$ при $\varepsilon \rightarrow 0$. Физически рассматриваемую задачу можно интерпретировать как задачу о потенциальной яме с бесконечно высокими стенками (см. [1], § 22), когда на дне ямы присутствует узкий конечный всплеск.

Заметим, что если вопрос о спектре оператора (1) в $\mathbb{R}^{n}$ заменой переменных $y=(x-$ $\left.x_{0}\right) \varepsilon^{-1}$ сводится к задачам, рассмотренным в [1], [2], то совершенно иная ситуация имеет место при изучении оператора (1) в ограниченной области. Легко видеть, что в данном случае указанная выше замена переменных не сводит задачу ни к ранее исследованной, ни к более простой задаче.

Целью настояшей работы является выяснение асимптотического поведения собственных значений оператора $\mathcal{H}_{\varepsilon}$ при $\varepsilon \rightarrow 0$. Подчеркнем, что, в отличие от работ [1]-[5], здесь рассматривается возмушение, которое носит сингулярньй характер и потому не может быть исследовано с помощью методик цитированных работ. Поэтому при построении асимптотик используется метод согласования асимптотических разложений [6]-[10]. Заметим также, что рассматриваемая задача не сводится и к схожим по постановке задачам с концентрированной массой (см., например, [11]).

В настоящей работе в явном виде построены первые члены асимптотических разложений собственных значений и собственных функций оператора $\mathcal{H}_{\varepsilon}$ по параметру $\varepsilon$. Показано, что эти первые поправки полностью определяются положением центра сжатия $x_{0}$ потенциала в $(1)$, а также его средним значением, причем последнее также определяет и знак первой поправки.

\section{2. ФОРМУЛИРОВКА ОСНОВНОГО РЕЗУЛЬТАТА}

Через $\mathcal{H}_{0}$ обозначим оператор $-\Delta$ в области $\Omega$ с краевым условием Дирихле на гранище. Пусть $\lambda_{0}$ - простое собственное значение оператора $\mathcal{H}_{0}, \psi_{0}$ - соответствующая нормированная в $L_{2}(\Omega)$ собственная функция.

Используя схему доказательств, примененную в [8], [12], [13], можно показать, что при $\varepsilon \rightarrow 0$ собственные значения и собственные функции оператора $\mathcal{H}_{\varepsilon}$ сходятся к собственным значениям и соответствующим собственным функциям оператора $\mathcal{H}_{0}$. Кроме того, к $\lambda_{0}$ сходится простое собственное значение $\lambda^{\varepsilon}$ оператора $\mathcal{H}_{\varepsilon}$, а соответствующая собственная функция $\psi^{\varepsilon}$ сходится к $\psi_{0}$ в норме $H^{1}(\Omega)$. Обозначим

$$
\langle V\rangle=\int_{\mathbb{R}^{n}} V(t) d t .
$$

Основной результат работы формулируется в виде следующего утверждения.

Теорема. Собственное значение $\lambda^{\varepsilon}$ оператора $\mathcal{H}_{\varepsilon}$, сходящееся $к$ простому собственному значению $\lambda_{0}$ оператора $\mathcal{H}_{0}$, имеет асимптотику

$$
\begin{aligned}
& \lambda^{\varepsilon}=\lambda_{0}+\varepsilon^{n} \lambda_{n}+O\left(\mu_{n}(\varepsilon)\right), \\
& \lambda_{n}=\psi_{0}^{2}\left(x_{0}\right)\langle V\rangle
\end{aligned}
$$


əде $\mu_{1}(\varepsilon)=\varepsilon^{3 / 2}, \quad \mu_{2}(\varepsilon)=\varepsilon^{3}|\ln \varepsilon|^{1 / 2}, \quad \mu_{3}(\varepsilon)=\varepsilon^{7 / 2}$.

ЗАМЕчАниЕ 1. В настоящей работе построены и асимптотики собственных функций. Однако представляется более удобным сформулировать соответствующее утверждение ниже (см. приведенную в разделе 5 лемму).

ЗАМЕчАниЕ 2. Подчеркнем, что асимптотики собственных значений, приводимые в теореме, не являются равномерными по номеру собственного значения. Не равномерны по номеру и асимптотики собственных функций, описанные в сформулированной в разделе 5 лемме.

Используя методику доказательства теоремы, можно получить полные асимптотические разложения собственных значений и собственных функций оператора $\mathcal{H}_{\varepsilon}$. В настоящей работе мы строим и обосновываем только первые члены асимптотических разложений, демонстрируя на их примере все идеи, необходимые для построения полных асимптотик. Построение полных асимптотик ничем не отличается от доказательства приведенной выше теоремы, но оно достаточно громоздкое с технической точки зрения. При получении строгой оценки остатка в асимптотиках (2) мы также стремились минимизировать число технических деталей, поэтому оценка остатка в (2) не является наилучшей по порядку.

Отметим, что нестрого формулу (3) можно получить следуюшим образом. Рассматривая потенциал в (1) как обобшенную функцию, мы можем представить его в виде

$$
V\left(\frac{x-x_{0}}{\varepsilon}\right)=\varepsilon^{n}\langle V\rangle \delta\left(x-x_{0}\right)+\cdots,
$$

где $\delta\left(x-x_{0}\right)$ - дельта-функция. Формально используя теперь формулу (38.6) из гл. 6 , $\S 38$ книги [1], получаем

$$
\lambda_{\varepsilon}=\lambda_{0}+\varepsilon^{n}\langle V\rangle \int_{\Omega} \psi_{0}^{2}(x) \delta\left(x-x_{0}\right) d x+\cdots=\lambda_{0}+\varepsilon^{n}\langle V\rangle \psi_{0}^{2}\left(x_{0}\right)+\cdots,
$$

что совпадает с первыми членами в (2). Данный подход, разумеется, не может рассматриваться как строгое математическое доказательство, так как использованная формула для первой поправки может быть применена, вообше говоря, лишь в случае регулярных возмушений (см. [14], гл. $2, \S 3$ ). Кроме того, подобный подход приводит к неверной асимптотике для собственных функций, так как формула (38.8) из гл. $6, \S 38$ [1] для первой поправки к собственной функции с точностью до слагаемого вида $C \psi_{0}(x)$ является разложением по собственным функциям оператора $\mathcal{H}_{0}$ функции $\psi_{n}$ из приведенной в разделе 5 леммы (что нетрудно проверить, умножая уравнение для $\psi_{n}$ на собственную функцию оператора $\mathcal{H}_{0}$, соответствуюшую $m$-му собственному значению, и интегрируя затем по частям). Функция $\psi_{n}$ имеет особенность в точке $x_{0}$, совпадающую с особенностью функции Грина для оператора Лапласа (см. ниже (12), (31)). В то же время, как следует из леммы, первая поправка к собственной функции помимо $\psi_{n}$ включает в себя еше одно слагаемое, причем данная сумма вышеупомянутой особенности не имеет. 
При доказательстве теоремы нам удобно будет рассматривать собственные значения и собственные функции операторов $\mathcal{H}_{\varepsilon}$ и $\mathcal{H}_{0}$ как собственные элементы следующих краевых задач:

$$
\begin{gathered}
-\Delta \psi^{\varepsilon}+V\left(\frac{x-x_{0}}{\varepsilon}\right) \psi^{\varepsilon}=\lambda^{\varepsilon} \psi^{\varepsilon}, \quad x \in \Omega, \quad \psi^{\varepsilon}=0, \quad x \in \partial \Omega \\
-\Delta \psi_{0}=\lambda_{0} \psi_{0}, \quad x \in \Omega, \quad \psi_{0}=0, \quad x \in \partial \Omega
\end{gathered}
$$

Статья построена следующим образом. Раздел 3 посвящен построению асимптотических разложений в условиях сформулированной теоремы в случае $n=1$. Построение в двумерном и трехмерном случаях проведено в разделе 4. Раздел 5 посвяшен строгому обоснованию асимптотических разложений, т.е. получению оценки остатка асимптотических разложений.

\section{3. ПОСТРОЕНИЕ АСИМПТОТИК В ОДНОМЕРНОМ СЛУЧАЕ}

В настоящем разделе будут формально построены первые члены асимптотик собственных значений и соответствуюших собственных функций задачи (4) в одномерном случае в условиях теоремы.

Сходимости $\lambda^{\varepsilon} \rightarrow \lambda_{0}, \psi^{\varepsilon} \rightarrow \psi_{0}$ означают, что главными членами асимптотических разложений $\lambda^{\varepsilon}$ и $\psi^{\varepsilon}$ являются соответственно $\lambda_{0}$ и $\psi_{0}$. Следуюшие члены асимптотических разложений $\lambda^{\varepsilon}$ и $\psi^{\varepsilon}$ будем строить так, чтобы эти асимптотики при подстановке в (4) давали достаточно малую невязку. Так как возмушающий потенциал в уравнении (4) сосредоточен на малом интервале и фактически зависит от "растянутой" переменной $\xi=\left(x-x_{0}\right) / \varepsilon$, то, следуя методу согласования асимптотических разложений [6], асимптотику собственной функции $\psi^{\varepsilon}$ в малой окрестности точки $x_{0}$ будем искать в виде ряда с коэффициентами, зависящими от переменной $\xi$ (внутреннее разложение). Вне окрестности точки $x_{0}$ асимптотику $\psi^{\varepsilon}$ будем искать в виде внешнего разложения, зависящего от переменной $x$.

Асимптотику собственного значения будем строить в виде

$$
\lambda^{\varepsilon}=\lambda_{0}+\varepsilon \lambda_{1}+\cdots .
$$

Внешнее разложение для собственной функции $\psi^{\varepsilon}$ будем искать в следуюшем виде:

$$
\begin{array}{ll}
\psi_{\varepsilon}^{\mathrm{ex},-}(x)=\psi_{0}(x)+\varepsilon \psi_{1}^{-}(x)+\cdots, & x<x_{0}, \\
\psi_{\varepsilon}^{\mathrm{ex},+}(x)=\psi_{0}(x)+\varepsilon \psi_{1}^{+}(x)+\cdots, & x>x_{0} .
\end{array}
$$

Выясним теперь вид внутреннего разложения. Ясно, что $\psi_{0} \in C^{\infty}[0,1]$, следовательно, в окрестности точки $x_{0}$ функция $\psi_{0}$ разлагается в ряд Тейлора:

$$
\psi_{0}(x)=\psi_{0}\left(x_{0}\right)+\psi_{0}^{\prime}\left(x_{0}\right)\left(x-x_{0}\right)+\frac{1}{2} \psi_{0}^{\prime \prime}\left(x_{0}\right)\left(x-x_{0}\right)^{2}+\mathcal{O}\left(\left(x-x_{0}\right)^{3}\right) .
$$

Переписывая теперь данное разложение в переменных $\xi$, видим, что при $x \rightarrow x_{0}$ вьполнено равенство

$$
\psi_{0}(x)=\psi_{0}\left(x_{0}\right)+\varepsilon \psi_{0}^{\prime}\left(x_{0}\right) \xi+\frac{1}{2} \varepsilon^{2} \psi_{0}^{\prime \prime}\left(x_{0}\right) \xi^{2}+O\left(\varepsilon^{3} \xi^{3}\right)
$$


Отсюда согласно методу согласования асимптотических разложений выводим, что внутреннее разложение для $\psi^{\varepsilon}$ должно выглядеть следуюшим образом:

$$
\psi_{\varepsilon}^{\text {in }}(\xi)=v_{0}(\xi)+\varepsilon v_{1}(\xi)+\varepsilon^{2} v_{2}(\xi)+\cdots,
$$

причем должны быть выполнены соотношения

$$
v_{i}(\xi)=\frac{\psi_{0}^{(i)}\left(x_{0}\right)}{i !} \xi^{i}+o\left(|\xi|^{i}\right), \quad \xi \rightarrow \pm \infty, \quad i=0,1,2 .
$$

Подставим теперь (8) и (6) в уравнение (4), перейдем к переменным $\xi$ и соберем коэффициенты при одинаковых степенях $\varepsilon$. Такая процедура приводит к следуюшим дифференциальным уравнениям:

$$
v_{0}^{\prime \prime}(\xi)=0, \quad v_{1}^{\prime \prime}(\xi)=0, \quad v_{2}^{\prime \prime}(\xi)=\left(V(\xi)-\lambda_{0}\right) v_{0}(\xi), \quad-\infty<\xi<\infty .
$$

Полученные уравнения для $v_{i}$ и асимптотики (9) определяют функции $v_{i}$ :

$$
\begin{aligned}
& v_{0}(\xi) \equiv \psi_{0}\left(x_{0}\right), \quad v_{1}(\xi)=\psi_{0}^{\prime}\left(x_{0}\right) \xi+c_{1}, \\
& v_{2}(\xi)=-\frac{1}{2} \lambda_{0} \psi_{0}\left(x_{0}\right) \xi^{2}+\tilde{v}_{2}(\xi)+c_{2} \xi+c_{3}, \\
& \tilde{v}_{2}(\xi)=\psi_{0}\left(x_{0}\right) \int_{\mathbb{R}} G_{1}(\xi-t) V(t) d t,
\end{aligned}
$$

где $c_{i}$ - некоторые пока произвольные константы,

$$
G_{1}(t)=\left\{\begin{array}{l}
0, \quad t<0 \\
t, \quad t \geqslant 0
\end{array}\right.
$$

Функция $v_{2}$, очевидно, имеет следующее поведение на бесконечности:

$$
v_{2}(\xi)= \begin{cases}-\lambda_{0} \psi_{0}\left(x_{0}\right) \xi^{2} / 2+c_{2} \xi+c_{3}, & \xi \rightarrow-\infty \\ -\lambda_{0} \psi_{0}\left(x_{0}\right) \xi^{2} / 2+\left(c_{2}+\psi_{0}\left(x_{0}\right)\langle V\rangle\right) \xi+\left(c_{3}-\psi_{0}\left(x_{0}\right)\langle\xi V\rangle\right), & \xi \rightarrow+\infty\end{cases}
$$

С учетом полученных асимптотик, равенства (8) и уравнения (5), взятого в точке $x_{0}$, получаем

$$
\begin{aligned}
& \psi_{\varepsilon}^{\text {in }}(x)= \\
& \quad= \begin{cases}\sum_{i=0}^{2} \frac{\psi_{0}^{(i)}\left(x_{0}\right)}{i !}\left(x-x_{0}\right)^{i}+\varepsilon\left(c_{1}+c_{2}\left(x-x_{0}\right)\right)+\cdots, & \xi \rightarrow-\infty, \\
\sum_{i=0}^{2} \frac{\psi_{0}^{(i)}\left(x_{0}\right)}{i !}\left(x-x_{0}\right)^{i}+\varepsilon\left(c_{1}+\left(c_{2}+\psi_{0}\left(x_{0}\right)\langle V\rangle\right)\left(x-x_{0}\right)\right)+\cdots, & \xi \rightarrow+\infty .\end{cases}
\end{aligned}
$$

Отсюда в силу метода согласования асимптотических разложений следует, что функции $\psi_{1}^{ \pm}$должны удовлетворять соотношениям

$$
\begin{array}{ll}
\psi_{1}^{-}(x)=c_{1}+c_{2}\left(x-x_{0}\right)+o\left(\left|x-x_{0}\right|\right), & x \rightarrow x_{0}-0, \\
\psi_{1}^{+}(x)=c_{1}+\left(c_{2}+\psi_{0}\left(x_{0}\right)\langle V\rangle\right)\left(x-x_{0}\right)+o\left(\left|x-x_{0}\right|\right), & x \rightarrow x_{0}+0,
\end{array}
$$


которые фактически являются граничными условиями для функций $\psi_{1}^{ \pm}$:

$$
\psi_{1}^{-}\left(x_{0}\right)=\psi_{1}^{+}\left(x_{0}\right), \quad \frac{d}{d x} \psi_{1}^{-}\left(x_{0}\right)=\frac{d}{d x} \psi_{1}^{+}\left(x_{0}\right)+\psi_{0}\left(x_{0}\right)\langle V\rangle .
$$

Определим теперь функции $\psi_{1}^{ \pm}$. Для этого подставим $(6),(7)$ в (4) и соберем коэффициенты при первой степени $\varepsilon$ :

$$
\begin{aligned}
& -\frac{d^{2} \psi_{1}^{-}}{d x^{2}}=\lambda_{0} \psi_{1}^{-}+\lambda_{1} \psi_{0}, \quad x \in\left(0, x_{0}\right), \quad \psi_{1}^{-}(0)=0, \\
& -\frac{d^{2} \psi_{1}^{+}}{d x^{2}}=\lambda_{0} \psi_{1}^{+}+\lambda_{1} \psi_{0}, \quad x \in\left(x_{0}, 1\right), \quad \psi_{1}^{+}(1)=0 .
\end{aligned}
$$

В рассматриваемом здесь одномерном случае собственное значение $\lambda_{0}$ и собственная функция $\psi_{0}$ выписываются явно: $\lambda_{0}=\pi^{2} m^{2}, \psi_{0}(x)=\sqrt{2} \sin (\pi m x), m \in \mathbb{N}$. Учитывая данный факт, легко найти общее решение задачи (11):

$$
\psi_{1}^{-}=\frac{\lambda_{1}}{2 \lambda_{0}} x \psi_{0}^{\prime}+c_{4} \psi_{0}, \quad \psi_{1}^{+}=\frac{\lambda_{1}}{2 \lambda_{0}}(x-1) \psi_{0}^{\prime}+c_{5} \psi_{0}
$$

где $c_{4}, c_{5}$ - константы. Подставляя теперь данные равенства в краевые условия (10), приходим к системе

$$
\begin{gathered}
\lambda_{1} \psi_{0}^{\prime}\left(x_{0}\right)+2 \lambda_{0}\left(c_{4}-c_{5}\right) \psi_{0}\left(x_{0}\right)=0, \\
\lambda_{1} \psi_{0}\left(x_{0}\right)-2\left(c_{4}-c_{5}\right) \psi_{0}^{\prime}\left(x_{0}\right)=2 \psi_{0}\left(x_{0}\right)\langle V\rangle .
\end{gathered}
$$

Решая эту систему с использованием очевидного равенства $\left(\psi^{\prime}\left(x_{0}\right)\right)^{2}+\lambda_{0} \psi_{0}^{2}\left(x_{0}\right)=2 \lambda_{0}$, получаем формулу (3) для $n=1$ и

$$
c_{4}-c_{5}=-\frac{\psi_{0}\left(x_{0}\right) \psi_{0}^{\prime}\left(x_{0}\right)\langle V\rangle}{2 \lambda_{0}} .
$$

Функции $\psi_{1}^{ \pm}(x)$ определены с точностью до слагаемого $c_{6} \psi_{0}$. Константу $c_{6}$ выберем так, чтобы добиться равенства $c_{1} \equiv \psi_{1}^{ \pm}\left(x_{0}\right)=0$. Такое требование окончательно определяет вид функций $\psi_{1}^{ \pm}$:

$$
\begin{aligned}
& \psi_{1}^{-}(x)=\frac{\psi_{0}\left(x_{0}\right)\langle V\rangle}{2 \lambda_{0}}\left(x \psi_{0}\left(x_{0}\right) \psi_{0}^{\prime}(x)-x_{0} \psi_{0}(x) \psi_{0}^{\prime}\left(x_{0}\right)\right) \\
& \psi_{1}^{+}(x)=\frac{\psi_{0}\left(x_{0}\right)\langle V\rangle}{2 \lambda_{0}}\left((x-1) \psi_{0}\left(x_{0}\right) \psi_{0}^{\prime}(x)-\left(x_{0}-1\right) \psi_{0}(x) \psi_{0}^{\prime}\left(x_{0}\right)\right)
\end{aligned}
$$




\section{4. ПОСТРОЕНИЕ АСИМПТОТИК В ДВУМЕРНОМ И ТРЕХМЕРНОМ СЛУЧАЯХ}

В настоящем разделе будут построены формальные асимптотики собственного значения и соответствуюших собственных функций краевой задачи (4) в случаях $n=2,3$ в условиях сформулированной выше теоремы.

Как и в предыдушем разделе, для построения асимптотик мы будем применять метод согласования асимптотических разложений. Асимптотику собственного значения будем искать в виде

$$
\lambda^{\varepsilon}=\lambda_{0}+\varepsilon^{n} \lambda_{n}+\cdots .
$$

Внешнее разложение для собственной функции в окрестности точки $x_{0}$ будем строить следуюшим образом:

$$
\psi_{\varepsilon}^{\mathrm{ex}}(x)=\psi_{0}(x)+\varepsilon^{n} \psi_{n}(x)+\cdots, \quad x \in \Omega \backslash\left\{x_{0}\right\} .
$$

В окрестности точки $x_{0}=\left(x_{1}^{0}, \ldots, x_{n}^{0}\right)$ введем новые "растянутые" переменные $\xi=$ $\left(\xi_{1}, \ldots, \xi_{n}\right), \xi_{i}=\left(x_{i}-x_{i}^{0}\right) \varepsilon^{-1}$. Положим $r=\left|x-x_{0}\right|, \rho=|\xi|$.

Так как $\psi_{0} \in C^{\infty}(\bar{\Omega})$, то в окрестности точки $x_{0}$ функция $\psi_{0}(x)$ удовлетворяет равенству

$$
\psi_{0}(x)=P_{0}+P_{1}\left(x-x_{0}\right)+P_{2}\left(x-x_{0}\right)+O\left(r^{3}\right), \quad r \rightarrow 0,
$$

где $P_{i}$-однородные полиномы степени $i$. Отметим, что в обоих случаях $(n=2,3)$ в силу уравнения (5) справедливо равенство

$$
-\Delta P_{2}=\lambda_{0} P_{0} .
$$

Переписьвая (15) в переменных $\xi$, получаем, что при $r \rightarrow 0$ справедливо

$$
\psi_{0}(x)=P_{0}+\varepsilon P_{1}(\xi)+\varepsilon^{2} P_{2}(\xi)+O\left(\varepsilon^{3} \rho^{3}\right) .
$$

Отсюда в силу метода согласования асимптотических разложений следует, что внутреннее разложение для обоих случаев $(n=2,3)$ должно иметь вид

$$
\psi_{\varepsilon}^{\text {in }}(\xi)=v_{0}(\xi)+\varepsilon v_{1}(\xi)+\varepsilon^{2} v_{2}(\xi)+\cdots,
$$

причем функции $v_{i}$ должны удовлетворять соотношениям

$$
v_{j}(\xi)=P_{j}(\xi)+o\left(\rho^{j}\right), \quad \rho \rightarrow \infty, \quad j=0,1,2,
$$

где $P_{0}(\xi) \equiv P_{0}$. Подставим $(13),(17)$ в $(4)$, перейдем к переменным $\xi$ и, выписывая коэффициенты при одинаковых степенях $\varepsilon$, в обоих случаях $n=2,3$ получаем уравнения

$$
\begin{aligned}
& \Delta_{\xi} v_{0}=0, \quad \Delta_{\xi} v_{1}=0, \quad \xi \in \mathbb{R}^{n}, \\
& \Delta_{\xi} v_{2}=\left(V(\xi)-\lambda_{0}\right) v_{0}, \quad \xi \in \mathbb{R}^{n} .
\end{aligned}
$$

Легко видеть, что функции

$$
v_{0}(\xi)=P_{0}, \quad v_{1}(\xi)=P_{1}(\xi)
$$


удовлетворяют уравнениям (19) и условиям (18). Учитывая (16), проверяем, что функция

$$
\begin{aligned}
& v_{2}(\xi)=P_{2}(\xi)+\tilde{v}_{2}(\xi), \\
& \tilde{v}_{2}(\xi)=P_{0} \int_{\mathbb{R}^{n}} V(\eta) G_{n}(\xi-\eta) d \eta,
\end{aligned}
$$

где

$$
G_{2}(t)=\frac{1}{2 \pi} \ln |t|, \quad G_{3}(t)=-\frac{1}{4 \pi|t|},
$$

является решением уравнения (20). Из (23) следует, что функция $\tilde{v}_{2}$ имеет следуюшую асимптотику при $\rho \rightarrow \infty$ :

$$
\tilde{v}_{2}(\xi)=P_{0}\langle V\rangle G_{n}(\rho)+O\left(\rho^{-n+1}\right) .
$$

Из соотношений $(21),(22),(24)$ вытекает, что при $\rho \rightarrow \infty$

$$
\psi_{\varepsilon}^{\text {in }}(\xi)=\sum_{i=0}^{2} \varepsilon^{i} P_{i}(\xi)+\varepsilon^{2} P_{0}\langle V\rangle G_{n}(\rho)+\cdots .
$$

Переписьвая теперь данное равенство в переменных $x$ и следуя методу согласования асимптотических разложений, заключаем, что внешнее разложение при $r \rightarrow 0$ должно иметь следующую асимптотику:

$$
\begin{array}{ll}
\psi_{\varepsilon}^{\mathrm{ex}}(x)=\sum_{i=0}^{2} P_{i}\left(x-x_{0}\right)+\varepsilon^{2} \frac{P_{0}\langle V\rangle}{2 \pi} \ln r-\varepsilon^{2} c_{7} \ln \varepsilon+\cdots, & n=2, \\
\psi_{\varepsilon}^{\mathrm{ex}}(x)=\sum_{i=0}^{2} P_{i}\left(x-x_{0}\right)-\varepsilon^{3} \frac{P_{0}\langle V\rangle}{4 \pi r}+\cdots, & n=3,
\end{array}
$$

где $c_{7}=P_{0}\langle V\rangle /(2 \pi)$. Как видно из полученных соотношений, в случае $n=2$ в асимптотике внешнего разложения появляется член порядка $O\left(\varepsilon^{2} \ln \varepsilon\right)$. Это означает, что, вообше говоря, в двумерном случае во внешнем разложении придется ввести член такого же порядка. Вместе с тем, если ввести во внутреннем разложении дополнительное слагаемое $\varepsilon^{2} v_{2,1} \ln \varepsilon$ с асимптотикой

$$
v_{2,1}(\xi)=\frac{P_{0}\langle V\rangle}{2 \pi}+o(1), \quad \rho \rightarrow \infty,
$$

член порядка $O\left(\varepsilon^{2} \ln \varepsilon\right)$ исчезнет в (25). С учетом данного дополнительного слагаемого внутреннее разложение для собственной функции в двумерном случае выглядит так:

$$
\psi_{\varepsilon}^{\text {in }}(\xi)=v_{0}(\xi)+\varepsilon v_{1}(\xi)+\varepsilon^{2} v_{2}(\xi)+\varepsilon^{2} v_{2,1}(\xi) \ln \varepsilon+\cdots .
$$

Подставив $(28)$ и (13) в (4) и выписав коэффициенты при $\varepsilon^{2} \ln \varepsilon$, получаем уравнение для $v_{2,1}$ :

$$
\Delta_{\xi} v_{2,1}=0, \quad \xi \in \mathbb{R}^{2}
$$


Очевидно, что функция

$$
v_{2,1}(\xi) \equiv \frac{1}{2 \pi} P_{0}\langle V\rangle
$$

является решением уравнения (29) с асимптотикой (27). Подставляя в $(28)$ вместо $v_{j}$ и $v_{2,1}$ их асимптотики на бесконечности (см. (21)- (24), (30)) и переписывая последние в переменных $x$, получаем, что при $\rho \rightarrow \infty$ должно быть выполнено соотношение $(25)$ с $c_{7}=0$. Следуя методу согласования асимптотических разложений, из $(14),(25)$ и (26) с $c_{7}=0$ заключаем, что функция $\psi_{n}$ из (14) должна удовлетворять следуюшей асимптотике:

$$
\psi_{n}(x)=P_{0}\langle V\rangle G_{n}(r)+O(1), \quad r \rightarrow 0 .
$$

Подстановка (13) и (14) в (4) приводит к краевой задаче для $\psi_{n}$ :

$$
-\Delta \psi_{n}=\lambda_{0} \psi_{n}+\lambda_{n} \psi_{0}, \quad x \in \Omega \backslash\left\{x_{0}\right\}, \quad \psi_{n}=0, \quad x \in \partial \Omega .
$$

Сушествование решения данной задачи, имеющего асимптотику (31), доказывается достаточно стандартным образом (см., например, [6], гл. 3 и [13]). Выведем условие разрешимости данной задачи. Умножим уравнение $(32)$ на $\psi_{0}$ и проинтегрируем полученное равенство по области $\Omega_{d}=\Omega \backslash\left\{x:\left|x-x_{0}\right| \leqslant d\right\}, d$ - малое число:

$$
-\int_{\Omega_{d}} \psi_{0} \Delta \psi_{n} d x=\lambda_{0} \int_{\Omega_{d}} \psi_{0} \psi_{n} d x+\lambda_{n} \int_{\Omega_{d}} \psi_{0}^{2} d x .
$$

В левой части данного равенства проведем интегрирование по частям, тогда получим

$$
\int_{\left|x-x_{0}\right|=d}\left(\psi_{0} \frac{\partial \psi_{n}}{\partial r}-\psi_{n} \frac{\partial \psi_{0}}{\partial r}\right) d s=\lambda_{n} \int_{\Omega_{d}} \psi_{0}^{2} d x .
$$

Подставим в левую часть данного равенства формулы (15) и (31) и перейдем затем к пределу при $d \rightarrow 0$. В результате с учетом нормировки $\psi_{0}$ приходим к формуле (3). Функция $\psi_{n}$ определена с точностью до слагаемого $C \psi_{0}$. Конкретный выбор константы $C$ для дальнейших рассуждений несушествен; для определенности мы будем предполагать, что константа $C$ выбрана из условия $\int_{\Omega} \psi_{n} \psi_{0} d x=0$.

Таким образом, формальная асимптотика собственного значения имеет вид (13), где $\lambda_{n}$ определяется формулой (3).

\section{5. ОБОСНОВАНИЕ АСИМПТОТИЧЕСКИХ РАЗЛОЖЕНИЙ}

В настоящем разделе получена оценка остатка для асимптотических разложений, построенных в разделах 3,4 .

Лемма. Справедливо утвер ждение теоремьи.

Собственную функиию $\Psi_{\varepsilon}$, соответствующую собственному значению $\lambda^{\varepsilon}$, можсно выбрать так, что в норме $H^{1}(\Omega)$ она будет иметь следующую асимптоmuкy:

$$
\begin{aligned}
& \Psi_{\varepsilon}(x)=\psi_{0}(x)+\varepsilon^{n} \psi_{n}(x)+ \\
& \quad+\varepsilon^{2} \psi_{0}\left(x_{0}\right) \int_{\mathbb{R}^{n}} V(\eta)\left(G_{n}\left(\frac{x-x_{0}}{\varepsilon}-\eta\right)-G_{n}\left(\frac{x-x_{0}}{\varepsilon}\right)\right) d \eta+O\left(\mu_{n}(\varepsilon)\right),
\end{aligned}
$$


где $\psi_{1}(x)=\psi_{1}^{ \pm}(x)$ при $\pm\left(x-x_{0}\right)>0$, функиии $\psi_{1}^{ \pm}$определяются соотношениями (12), $\psi_{2}, \psi_{3}$ - решения краевой задачи (32) с асимптотикой (31).

ЗАмЕчаниЕ 3 . Асимптотическое разложение собственной функции $\Psi_{\varepsilon}(33)$ является суммой внешнего и внутреннего разложений, построенных в разделах 3,4 , из которой в соответствии с методом согласования асимптотических разложений были вычтены согласованные члены данных разложений:

$$
\begin{gathered}
\psi_{0}(x)+\varepsilon^{n} \psi_{n}(x)+v_{0}+\varepsilon v_{1}(\xi)+\varepsilon^{2} v_{2}(\xi)- \\
-\left(P_{0}+\varepsilon P_{1}(\xi)+\varepsilon^{2} P_{2}(\xi)+\varepsilon^{2} \psi_{0}\left(x_{0}\right) G_{n}(\xi)\langle V\rangle\right)=\psi_{0}(x)+\varepsilon^{n} \psi_{n}(x)+\varepsilon^{2} \hat{v}_{2}(\xi), \\
\hat{v}_{2}(\xi)=\psi_{0}\left(x_{0}\right) \int_{\mathbb{R}^{n}} V(\eta)\left(G_{n}(\xi-\eta)-G_{n}(\xi)\right) d \eta .
\end{gathered}
$$

ДОКАЗАТЕЛЬСТво ЛЕммЫ. Обозначим $\hat{\lambda}^{\varepsilon}=\lambda_{0}+\varepsilon^{n} \lambda_{n}, \phi_{\varepsilon}(x)=\psi_{0}(x)+\varepsilon^{n} \psi_{n}(x)+$ $\varepsilon^{2} \hat{v}_{2}(\xi)$. Начнем с одномерного случая. Покажем, что асимптотические разложения, построенные в разделе 3 , удовлетворяют краевой задаче (4) с точностью до достаточно малой невязки. Положим

$$
R_{\varepsilon}(x)=\varepsilon^{2} \psi_{0}\left(x_{0}\right)\langle t V(t)\rangle x, \quad \widehat{\psi}^{\varepsilon}(x)=\phi_{\varepsilon}(x)-R_{\varepsilon}(x) .
$$

Учитьвая определение функции $\psi_{1}$, нетрудно убедиться, что функция $\widehat{\psi}^{\varepsilon}(x)$ является элементом $H^{2}(\Omega)$ и обрашается в нуль на границе области $\Omega$. Прямыми вычислениями с учетом уравнений (11) проверяем, что функция

$$
f_{\varepsilon}=\left(\mathcal{H}_{\varepsilon}-\hat{\lambda}^{\varepsilon}\right) \widehat{\psi}^{\varepsilon}
$$

имеет следуюший вид:

$$
\begin{aligned}
f_{\varepsilon} & =-\sum_{i=1}^{3} f_{\varepsilon, i}, \\
f_{\varepsilon, 1}(x) & =\varepsilon^{2} \lambda_{1} \psi_{1}+\left(\hat{\lambda}^{\varepsilon}-V\left(\frac{x-x_{0}}{\varepsilon}\right)\right) R_{\varepsilon}, \\
f_{\varepsilon, 2}(x) & =-V\left(\frac{x-x_{0}}{\varepsilon}\right)\left(\psi_{0}(x)-\psi_{0}\left(x_{0}\right)+\varepsilon \psi_{1}\right), \\
f_{\varepsilon, 3}(x) & =\varepsilon^{2}\left(\hat{\lambda}^{\varepsilon}-V\left(\frac{x-x_{0}}{\varepsilon}\right)\right) \hat{v}_{2}(\xi) .
\end{aligned}
$$

Функция $\psi_{1}$ - непрерывна в $\bar{\Omega}$, а потому с учетом определения $R_{\varepsilon}$ получаем $\left\|f_{\varepsilon, 1}\right\|=$ $O\left(\varepsilon^{2}\right)$, где $\|\cdot\|$ - норма в $L_{2}(\Omega)$. Функция $V\left(\left(x-x_{0}\right) / \varepsilon\right)$ не равна нулю лишь в малой (порядка $O(\varepsilon)$ ) окрестности точки $x_{0}$. В этой окрестности в силу гладкости функции $\psi_{0}(x)$ верна равномерная по $\varepsilon$ и $x$ оценка

$$
\left|\psi_{0}(x)-\psi_{0}\left(x_{0}\right)\right| \leqslant C \varepsilon .
$$


Отсюда с учетом ограниченности и финитности функции $V$ и ограниченности функции $\psi_{1}$ (см. (12)) получаем

$$
\left\|f_{\varepsilon, 2}\right\|^{2} \leqslant C \varepsilon^{2}\left\|V\left(\frac{x-x_{0}}{\varepsilon}\right)\right\|^{2} \leqslant C \varepsilon^{3},
$$

откуда следует $\left\|f_{\varepsilon, 2}\right\|=O\left(\varepsilon^{3 / 2}\right)$. По определению функция $\hat{v}_{2}(\xi)$ ограниченная, а потому $\left\|f_{\varepsilon, 3}\right\|=O\left(\varepsilon^{2}\right)$. Следовательно,

$$
\left\|f_{\varepsilon}\right\|=O\left(\varepsilon^{3 / 2}\right)
$$

Собственное значение $\lambda^{\varepsilon}$ является простым полюсом резольвенты оператора $\mathcal{H}_{\varepsilon}$ (так как оператор самосопряжен, см. [14], гл. $5, \S 3.5)$. Используя данный факт, аналогично [8], [13] можно показать, что при $\lambda$, близких к $\lambda_{0}$, для всех $f \in L_{2}(\Omega)$ справедливо представление

$$
\left(\mathcal{H}_{\varepsilon}-\lambda\right)^{-1} f=\frac{\psi^{\varepsilon}}{\lambda^{\varepsilon}-\lambda} \int_{\Omega} \psi^{\varepsilon} f d x+u_{\varepsilon}
$$

где $\psi^{\varepsilon}$ - нормированная в $L_{2}(\Omega)$ собственная функция, соответствуюшая $\lambda^{\varepsilon}$, а функция $u_{\varepsilon}$ удовлетворяет оценке

$$
\left\|u_{\varepsilon}\right\|_{1} \leqslant C\|f\| .
$$

Здесь константа $C$ не зависит от $\varepsilon, \lambda$ и $f,\|\cdot\|_{1}$ - норма в $H^{1}(\Omega)$. Представление $(35)$ и оценка (36) верны для любой размерности.

Применяя теперь представление (35) к функции $f_{\varepsilon} \mathrm{c} \lambda=\hat{\lambda}^{\varepsilon}$, с учетом нормированности $\psi^{\varepsilon}$ и очевидного равенства $\widehat{\psi}^{\varepsilon}=\left(\mathcal{H}_{\varepsilon}-\hat{\lambda}^{\varepsilon}\right)^{-1} f_{\varepsilon}$ получаем очевидную оценку:

$$
\left\|\widehat{\psi}^{\varepsilon}-u_{\varepsilon}\right\| \leqslant \frac{\left\|f_{\varepsilon}\right\|}{\left|\lambda^{\varepsilon}-\hat{\lambda}^{\varepsilon}\right|}
$$

Так как норма $\left\|u_{\varepsilon}\right\|$ мала в силу (34), (36), а функция $\widehat{\psi}^{\varepsilon}$ по построению, очевидно, сходится к $\psi_{0}$ в $L_{2}(\Omega)$ при $\varepsilon \rightarrow 0$, то из (34), (37) немедленно следует:

$$
\left|\hat{\lambda}^{\varepsilon}-\lambda^{\varepsilon}\right| \leqslant C\left\|f_{\varepsilon}\right\|=O\left(\varepsilon^{3 / 2}\right),
$$

что доказывает теорему в одномерном случае. Далее, первое слагаемое правой части (35) является, очевидно, собственной функцией, соответствуюшей $\lambda^{\varepsilon}$. Обозначим эту собственную функцию через $\Psi_{\varepsilon}$. В силу представления $(35)$ для функции $f_{\varepsilon}$, оценок $(34),(36)$ и равенства $\left\|R_{\varepsilon}\right\|_{1}=O\left(\varepsilon^{2}\right)$ имеем

$$
\left\|\phi_{\varepsilon}-\Psi_{\varepsilon}\right\|_{1} \leqslant\left\|\widehat{\psi}^{\varepsilon}-\Psi_{\varepsilon}\right\|_{1}+\left\|R_{\varepsilon}\right\|_{1}=\left\|u_{\varepsilon}\right\|_{1}+\left\|R_{\varepsilon}\right\|_{1}=O\left(\varepsilon^{3 / 2}\right),
$$

что доказывает асимптотику (33) в одномерном случае.

В случаях $n=2,3$ доказательство в целом аналогично. Отличается лишь вид функции $R_{\varepsilon}$, так как в этом случае она более сложная и содержит большее число членов. 
Для того чтобы определить ее, необходимо ввести в рассмотрение следующие дополнительные функции. Положим

$$
\hat{v}_{3}(\xi)=\int_{\mathbb{R}^{n}} V(\eta) P_{1}(\eta)\left(G_{n}(\xi-\eta)-G_{n}(\xi)\right) d \eta .
$$

Функцию $\widehat{\psi}_{n+1}$ определим как гармоническую в $\Omega$, удовлетворяюшую однородному граничному условию Дирихле на границе и имеющую следующее поведение при $x \rightarrow x_{0}$ :

$$
\widehat{\psi}_{n+1}(x)=\left\langle P_{1} V\right\rangle G_{n}(r)+O(1) .
$$

В малой окрестности границы $\Omega$ введем координаты $(s, \tau)$, где $s$ - координаты на поверхности $\partial \Omega, \tau$ - расстояние от точки до $\partial \Omega$, измеренное в направлении внутренней нормали. Пусть $\chi(x)$ - бесконечно дифференцируемая срезаюшая функция, равная нулю вне окрестности $\partial \Omega$, в которой определены координаты $(s, \tau)$, и единице в некоторой меньшей окрестности. Также предполагается, что носители $\chi(x)$ и $V\left(\left(x-x_{0}\right) / \varepsilon\right)$ не пересекаются для всех достаточно малых $\varepsilon$. Положим

$$
g(s, \varepsilon):=\varepsilon^{2} \int_{\mathbb{R}^{n}} V(\eta)\left(P_{0}+\varepsilon P_{1}(\eta)\right)\left(G_{n}(\xi-\eta)-G_{n}(\xi)\right) d \eta, \quad x \in \partial \Omega .
$$

Нетрудно убедиться, что

$$
g(s, \varepsilon)=\varepsilon^{n+1} \tilde{g}(s, \varepsilon),
$$

где $\tilde{g}(s, \varepsilon)$ - бесконечно дифференцируемая по $s \in \partial \Omega$ и $\varepsilon \in\left[0, \varepsilon_{0}\right]$ функция, $\varepsilon_{0}$ - достаточно малое число. Определим функцию $R_{\varepsilon}$ следующим образом:

$$
R_{\varepsilon}(x):=\varepsilon^{3} \hat{v}_{3}(\xi)+\varepsilon^{n+1} \widehat{\psi}_{n+1}(x)-g(s, \varepsilon) \chi(x) .
$$

Используя данную функцию $R_{\varepsilon}$, определим $\widehat{\psi}^{\varepsilon}$ и $f_{\varepsilon}$ так же, как и в одномерном случае. Прямыми вычислениями проверяется, что функция $\widehat{\psi}^{\varepsilon}(x)$ является элементом $H^{2}(\Omega)$ и удовлетворяет однородному краевому условию Дирихле на границе $\partial \Omega$, а функция $f_{\varepsilon}$ представляется в виде

$$
\begin{aligned}
f_{\varepsilon} & =-\sum_{i=1}^{3} f_{\varepsilon, i}, \\
f_{\varepsilon, 1}(x) & =\varepsilon^{2 n} \lambda_{n} \psi_{n}(x)-\left(\Delta-V\left(\frac{x-x_{0}}{\varepsilon}\right)+\hat{\lambda}^{\varepsilon}\right) g(x, \varepsilon) \chi(x), \\
f_{\varepsilon, 2}(x) & =-V\left(\frac{x-x_{0}}{\varepsilon}\right)\left(\psi_{0}(x)-P_{0}-P_{1}\left(x-x_{0}\right)+\varepsilon^{n} \psi_{n}(x)\right), \\
f_{\varepsilon, 3} & =\left(\hat{\lambda}^{\varepsilon}-V\left(\frac{x-x_{0}}{\varepsilon}\right)\right)\left(\varepsilon^{n+1} \widehat{\psi}_{n+1}+\varepsilon^{2} \hat{v}_{2}(\xi)+\varepsilon^{3} \hat{v}_{3}(\xi)\right) .
\end{aligned}
$$

Учитывая равенство (38) и принадлежность функции $\psi_{n}$ к $L_{2}(\Omega)$, нетрудно проверить, что $\left\|f_{\varepsilon, 1}\right\|=O\left(\varepsilon^{n+1}\right)$. Функция $V\left(\left(x-x_{0}\right) / \varepsilon\right)$ не равна нулю лишь в малой окрестности точки $x_{0}$, где функция $\psi_{0}(x)-P_{0}-P_{1}(x)$ оценивается по модулю величиной порядка $O\left(\varepsilon^{2}\right)$, а потому верно $\left\|f_{\varepsilon, 2}\right\|=O\left(\varepsilon^{n / 2+2}\right)$. Используя очевидные равенства $\hat{v}_{i}(\xi)=O\left(|\xi|^{-n+1}\right)$ при $\xi \rightarrow \infty$ и принадлежность $\widehat{\psi}_{n+1}$ к $L_{2}(\Omega)$, нетрудно убедиться, что $\left\|f_{\varepsilon, 3}\right\|=O\left(\mu_{n}(\varepsilon)\right)$. Дальнейшее доказательство проводится аналогично одномерному случаю. При этом для доказательства асимптотики собственной функции следует лишь учесть, что $\left\|R_{\varepsilon}\right\|_{1}=O\left(\varepsilon^{n / 2+2}\right)$. Лемма полностью доказана. 
Благодарности. Авторы выражают благодарность Р. Р. Гадыльшину за постоянное внимание к работе и полезные замечания. Также авторы благодарят П. Экснера за обсуждение результатов работы. Д. И. Борисов благодарит за гостеприимство Университет Штутгарта (Германия), где была выполнена часть этой работы. Работа выполнена при частичной финансовой поддержке программы "Ведушие научные школы" (НШ-1446.2003.1).А.Р. Бикметов поддержан грантом РФФИ (№ 05-01-97912-р_агидель), Д. И. Борисов - программой “Университеты России” (УР 04.01.484).

\section{Список литературы}

[1] Л. Д. Ландау, Е. М. Лившиц. Квантовая механика. Нерелятивистская теория. М.: Наука, 1989.

[2] B. Simon. Ann. Phys. 1976. V. 97. P. 279-288; M. Klaus. Ann. Phys. 1977. V. 108. P. 288-300; R. Blankenbecler, M. L. Goldberger, B. Simon. Ann. Phys. 1977. V. 108. P. 69-78; M. Klaus, B. Simon. Ann. Phys. 1980. V. 130. P. 251-281.

[3] Д. Е. Пелиновский, К. Салем. ТМФ. 2000. Т. 122. С. 118-128; P. Zhevandrov, A. Merzon. Asymptotics of eigenfunctions in shallow potential wells and related problems. In: Asymptotic Methods for Wave and Quantum Problems. AMS Transl. Ser. 2. Adv. Math. Sci. V. 208. Ed. M. V. Karasev. Providence, RI: AMS, 2003. P. 235-284.

[4] F. Bentosela, R. M. Cavalcanti, P. Exner, V.A. Zagrebanov. J. Phys. A. 1999. V. 32. P. 3029-3039.

[5] Р. Р. Гадыльииин. ТМФ. 2002. Т. 132. С. 97-104; math-ph/0202015; 2004. Т. 138. C. 41-54; math-ph/0208025.

[6] A. М. Ильин. Согласование асимптотических разложений решений краевых задач. М.: Наука, 1989.

[7] В. Г. Мазъя, С. А. Назаров, Б. А. Пламеневский. Изв. АН СССР. Сер. матем. 1984. Т. 48. C. $347-371$.

[8] Р. Р. Гадыльшин. Диффер. уравнения. 1986. Т. 22. С. 640-652.

[9] Р. Р. Гадыльиин. Матем. заметки. 1992. Т. 52. № 4. С. 42-55.

[10] M. Yu. Planida. Comptes Rendus Mecanique. 2003. V. 331. P. 531-536.

[11] E. Sánchez-Palencia. Perturbation of eigenvalues in thermoelasticity and vibration of systems with concentrated masses. In: Trends and Applications of Pure Mathematics to Mechanics. Proc. of Symp. at Ecole Polytechnique (Palaiseau, France, November 28 - December 2, 1983). Lect. Notes Phys. V. 195. Eds. P. G. Ciarlet, M. Roseau. Berlin: Springer, 1984. P. 346-368; О.А. Олейник, Г.А. Иосифьян, А.С. Шамаев. Математические задачи теории сильно неоднородных упругих сред. М.: Изд-во МГУ, 1990; Ю. Д. Головатий, И. А. Головач. Вісн. Львів. ун-ту. Сер. мех.-матем. 1997. № 48. С. 88-99.

[12] М. Ю. Планида. Матем. заметки. 2001. Т. 71. № 6. С. 867-877.

[13] М. Ю. Планида. ЖВМиМФ. 2003. Т. 43. С. 422-432.

[14] Т. Като. Теория возмушений линейных операторов. М.: Мир, 1972.

Поступила в редакцию 19.V.2004 г., после доработки 3.V.2005 г. 